\title{
Papiloma escamoso em adolescente com deficiência intelectual: um relato de caso
}

\author{
Squamous papilloma in an adolescent with intellectual disability: a case report \\ Papiloma escamoso en un adolescente con discapacidad intelectual: informe de un caso
}

Recebido: 02/12/2021 | Revisado: 08/12/2021 | Aceito: 17/12/2021 | Publicado: 01/01/2022

\author{
William José e Silva Filho \\ ORCID: https://orcid.org/0000-0002-2117-3352 \\ Universidade Federal de Sergipe, Brasil \\ E-mail: williamjsfilho10@gmail.com \\ Bruno Natan Santana Lima \\ ORCID: https://orcid.org/0000-0003-2828-2129 \\ Universidade Federal de Sergipe, Brasil \\ E-mail: brunonatanufs@gmail.com \\ Graziane Ribeiro Couto \\ ORCID: https://orcid.org/0000-0002-4443-7867 \\ Universidade Federal de Sergipe, Brasil \\ E-mail: grazianeribeiro.couto@gmail.com \\ Gustavo Almeida Souza \\ ORCID: https://orcid.org/0000-0003-1040-1273 \\ Universidade Federal de Sergipe, Brasil \\ E-mail: gustavosouzabmf@gmail.com \\ Gabriela Mancia de Gutierrez \\ ORCID: https://orcid.org/0000-0003-1538-5823 \\ Universidade Federal de Sergipe, Brasil \\ E-mail: gabrielamancia@ hotmail.com
}

\begin{abstract}
Resumo
O objetivo desse estudo foi relatar um caso clínico de papiloma escamoso oral, abordando seus aspectos clínicos, histopatológicos e conduta clínica de tratamento. Paciente do sexo feminino, 16 anos de idade, com deficiência intelectual, chegou ao Centro de Especialidades Odontológicas com queixa principal de "dentes estragados". No exame clínico intraoral foi observada, além de unidades dentárias comprometidas com a doença cárie, uma lesão em ápice de língua, de aspecto papilar, medindo aproximadamente $0,5 \mathrm{~cm}$, coloração normal de mucosa, séssil de bordos bem delimitados. Devido ao íntimo contato com os incisivos centrais inferiores que se apresentavam-se apinhados, foi sugerido como hipótese diagnóstica de fibroma traumático. A biopsia excisional foi realizada e o resultado do exame anatomopatológico foi de papiloma escamoso oral. Foi realizada orientação a família da necessidade de acompanhamento periódicos para identificação de futuras possíveis lesões orais, encaminhamento da paciente ao Ginecologista, já que a mesma nunca havia ido ao especialista, e segundo a mãe a filha não havia iniciado a vida sexual, mas já havia sido imunizada contra o HPV. Salienta-se que todas as lesões de boca persistente devem ser biopsiadas, pois lesões que aparentemente inofensivas podem ter diagnóstico que exigem uma abordagem mais criteriosa.
\end{abstract}

Palavras-chave: Infecção por HPV; Diagnóstico bucal; Medicina bucal.

\begin{abstract}
The aim of this study was to report a clinical case of oral squamous papilloma, addressing its clinical and histopathological aspects and clinical treatment approach. A 16-year-old female patient with an intellectual disability arrived at the Dental Specialties Center with the main complaint of "damaged teeth". In the intraoral clinical examination, in addition to dental units compromised with caries disease, a lesion in the apex of the tongue, with a papillary aspect, measuring approximately $0.5 \mathrm{~cm}$, normal color of the mucosa, sessile with well-defined edges was observed. Due to the close contact with the lower central incisors that were crowded, it was suggested as a diagnostic hypothesis of traumatic fibroma. Excisional biopsy was performed and the result of the anatomopathological examination was oral squamous papilloma. The family was instructed on the need for periodic follow-up to identify possible future oral lesions, referral of the patient to the Gynecologist, since she had never been to the specialist, and according to the mother, the daughter had not started sex, but had already been immunized against HPV. It should be noted that all persistent mouth lesions should be biopsied, as lesions that are apparently harmless can be diagnosed and require a more careful approach.
\end{abstract}

Keywords: Papillomavirus Infections; Oral diagnosis; Oral medicine. 
Research, Society and Development, v. 11, n. 1, e3411124292, 2022

(CC BY 4.0) | ISSN 2525-3409 | DOI: http://dx.doi.org/10.33448/rsd-v11i1.24292

\section{Resumen}

El objetivo de este estudio fue reportar un caso clínico de papiloma escamoso oral, abordando sus aspectos clínicos, histopatológicos y abordaje clínico del tratamiento. Una paciente de 16 años con discapacidad intelectual llegó al Centro de Especialidades Dentales con el principal síntoma de "dientes dañados". En el examen clínico intraoral, además de las unidades dentarias comprometidas con enfermedad de caries, se observó una lesión en el ápice de la lengua, de aspecto papilar, de aproximadamente $0,5 \mathrm{~cm}$, color normal de la mucosa, sésil con bordes bien definidos. Debido al estrecho contacto con los incisivos centrales inferiores que estaban apiñados, se sugirió como hipótesis diagnóstica de fibroma traumático. Se realizó biopsia excisional y el resultado del examen anatomopatológico fue papiloma escamoso oral. Se instruyó a la familia sobre la necesidad de seguimiento periódico para identificar posibles lesiones bucales futuras, derivación de la paciente al ginecólogo, ya que nunca había ido al especialista, y según la madre, la hija no había iniciado relaciones sexuales, pero ya había sido inmunizado contra el VPH. Cabe señalar que todas las lesiones bucales persistentes deben ser biopsiadas, ya que las lesiones aparentemente inofensivas pueden diagnosticarse y requieren un abordaje más cuidadoso.

Palabras clave: Infecciones por Papillomavirus, Diagnóstico bucal, Medicina oral.

\section{Introdução}

O grupo de papilomavírus humanos (HPV) pertence à família Papillomaviridae e seu ciclo de vida está diretamente ligado à diferenciação das células epiteliais do hospedeiro (Ferraro et al., 2011). Formado por dNA helicoidal de fita dupla e com capsídeo proteico icosaédrico, os HPVs replicam seus genomas dentro do núcleo das células do hospedeiro, que o tornam partículas intracelulares obrigatórias, com tropismo específico para os ceratinócitos. Logo, a cavidade oral por ser revestida por uma mucosa constituída por epitélio escamoso estratificado e lâmina própria com tecido conjuntivo denso, torna-se sítio de infecção (Ferraro, 2011; Gleason et al., 2016).

O HPV infecta o epitélio em seu estado basal mantendo a transcrição e replicação de seu DNA em níveis basais, produzindo lesões consideradas comuns à associação ao HPV, com características papilomatosas e verrucosas hiperplásicas na pele e na mucosa, como proliferações exofíticas de curso benigno, sendo ainda relatado mais de 100 tipos de HPV já identificados, dos quais 24/30 já foram localizados na cavidade oral (Neville, 2016; Ferraro, 2011; Gleason, 2016; Piña et al., 2019). As lesões benignas relacionadas ao HPV que acometem a cavidade oral se apresentam com uma grande diversidade em seus aspectos clínicos e histopatológicos, logo seu correto diagnóstico representa um desafio para os profissionais (Betz., 2019).

Dentre as lesões orais, quatro são reconhecidas pela Organização Mundial da Saúde (OMS) como relacionadas ao HPV: papiloma de células escamosas, condiloma acuminado, verruga vulgar e hiperplasia epitelial multifocal (Piña et al., 2019). O papiloma escamoso da mucosa oral é a lesão papilar mais comum, representando 2,5\% de todas as lesões na cavidade oral (González et al., 2016). Pode acometer qualquer região da cavidade oral, no entanto as lesões clínicas do HPV são mais frequentemente observadas na mucosa labial, língua, mucosa jugal, gengiva, bem como no palato mole e palato duro (Neville., 2016).

A apresentação clínica do papiloma escamoso oral é descrita como um crescimento exofítico com superfície rugosa, semelhante à couve-flor e, dependendo do grau de queratinização da lesão, pode ser branco, rosa e/ou avermelhada, pediculada ou séssil (Testi et al., 2016). Por apresentar ausência de sintomatologia, baixa taxa de virulência e infecciosidade baixa, seu tratamento de escolha consiste na remoção cirúrgica (González et al., 2016; Orenuga et al., 2018), mas também pode-se lançar mão do uso de crioterapia e da terapia fotodinâmica antimicrobiana (aPDT) (Orrú, et al., 2019). A biópsia e exame histopatológico possibilita diferenciar o papiloma escamoso frente a outras lesões. Seu prognóstico é bom, porém, é importante manter um controle periódico de acompanhamento por um período de cinco anos devido a possibilidade de recidiva (González et al., 2016; Orenuga et al., 2018). 
O papiloma escamoso oral é uma lesão relacionada ao HPV, que é contagioso e pode ser transmitido por ato sexual, sendo considerado uma doença sexualmente transmissível (DST) (Castro et al., 2004). A contaminação via objetos é rara, porém a transmissão vertical da mãe para o bebê durante a gravidez, no parto ou na amamentação também é causa dessa infecção (Castro et al., 2004). A lesão quando acomete a cavidade oral de crianças e adolescentes deve se atentar na investigação da causa da infecção pelo HPV, sendo importante para detecção de possíveis casos de abuso sexual.

O objetivo do presente estudo é relatar um caso clínico de papiloma escamoso oral em língua, abordando seus aspectos clínicos, histopatológicos e conduta clínica de tratamento.

\section{Metodologia}

O presente trabalho trata-se de um relato de caso, sendo o mesmo, descritivo e qualitativo, sendo orientado e confeccionado a partir das diretrizes tradicionais de estratégia de pesquisa, sendo ainda, apoiado por técnicas de observação e descrição clara, estas, apoiadas a partir de revisão de literatura e observação direta. Dessa forma, o referido trabalho integra-se como um método abrangente de coleta de materiais e análise de dados, compreendendo uma descrição clara e discussão de fatores que transitam desde o diagnóstico até o tratamento da condição apresentada (Pereira et al., 2018). Assim, visando obedecer aos aspectos éticos a respeito de pesquisas e testes com seres humanos, e de acordo com a Declaração de Helsinque, foi realizada a assinatura pela responsável da paciente, sendo, portanto, assinado o termo de consentimento livre e esclarecido (TCLE), desta forma, autorizado a utilização dos dados aqui apresentados do seu caso clínico e documentação fotográfica explicitada em nosso estudo (Yoshida., 2007).

\section{Relato de Caso}

Paciente do sexo feminino, 16 anos, leucoderma, compareceu ao Centro de Especialidade Odontológicas de Boquim, Sergipe, com queixa principal de "dentes estragados". Na anamnese, a mãe da paciente relatou que a filha possuía deficiência intelectual, falava pouco, mas com bom entendimento e comportamento.

Ao realizar o exame clínico intrabucal foi observado, restos radiculares das unidades dentárias 36 e 46 , ausência da unidade 12, além de uma lesão em ápice de língua, de aspecto papilar, medindo aproximadamente 0,5 cm, normocrômica, séssil e de bordos bem delimitados (Figura 1).

Figura 1: Aspecto clínico inicial da lesão em ápice de língua.

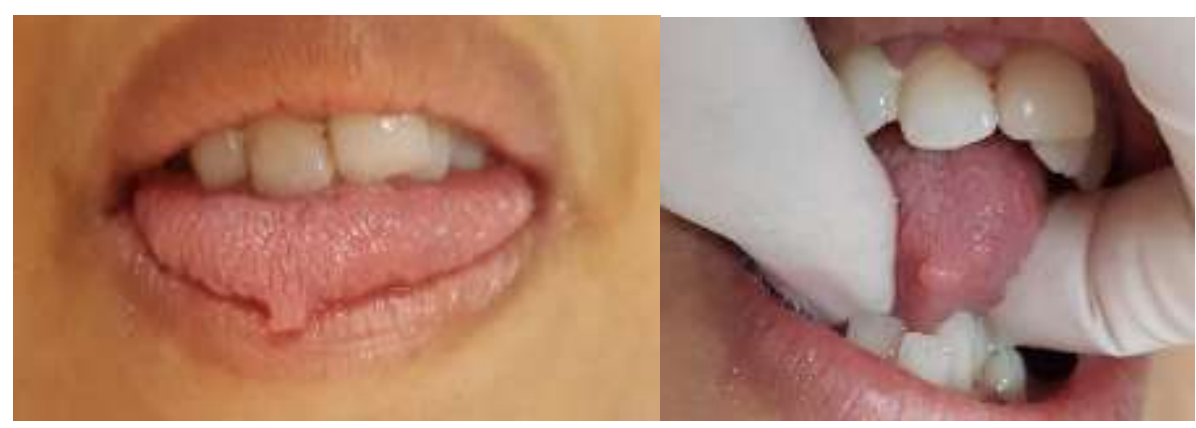

Fonte: Autores.

Devido ao íntimo contato com os incisivos centrais inferiores apinhados, foi sugerido como hipótese diagnóstica de fibroma traumático.

Solicitada radiografia panorâmica para investigação de agenesia da unidade 12 e observar as condições gerais das unidades acometidas com a doença cárie para estabelecer o plano de tratamento (Figura 2). 
Research, Society and Development, v. 11, n. 1, e3411124292, 2022

(CC BY 4.0) | ISSN 2525-3409 | DOI: http://dx.doi.org/10.33448/rsd-v11i1.24292

Figura 2: Radiografia panorâmica indicando agenesia da unidade dentária 12 e restos radiculares das unidades dentárias 36 e 46.

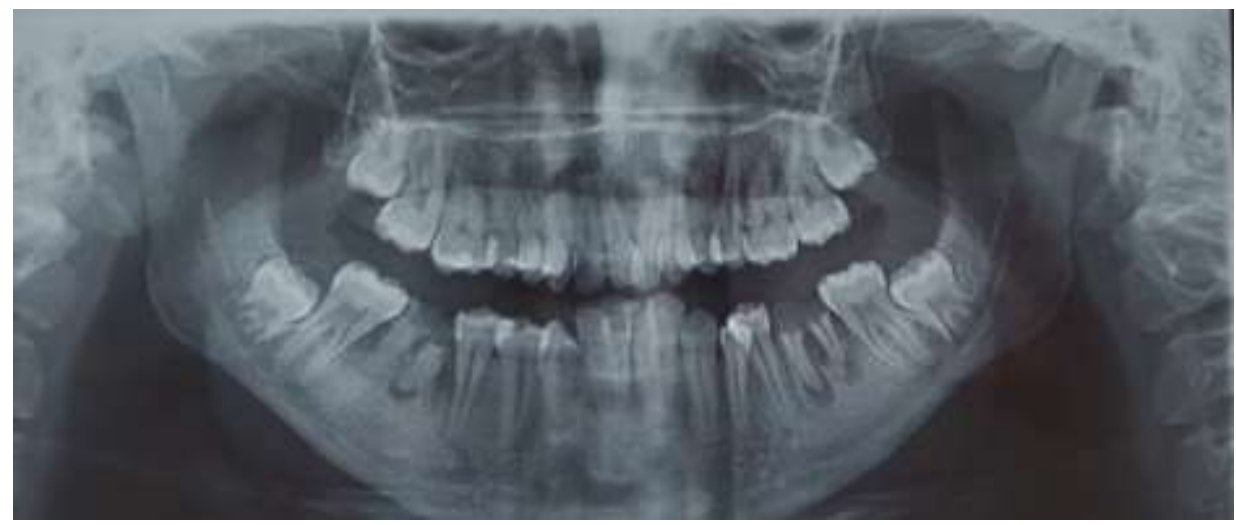

Fonte: Autores.

Optou-se por realizar a biópsia excisional da lesão no mesmo tempo cirúrgico de uma exodontia previamente planejada da unidade 46, por questões de conveniência e conforto da paciente já que a mesma já teria sido submetida a anestesia por bloqueio do nervo alveolar inferior. Com uma pinça allis foi realizada a apreensão da lesão e as incisões, com uso da lâmina de bisturi, ocorreram ao longo da base da lesão de forma elíptica envolvendo-a. Após os procedimentos cirúrgicos suturas simples foram realizadas. A paciente foi orientada quanto a dieta e cuidados pós operatórios.

O exame anatomopatológico exibiu lesão constituída por eixo fibrovascular papilífero, com epitélio escamoso estratificado exibindo papilomatose, acantose e hiperceratose, sem atipias e com presença de halos claros perinucleares superficiais (Figura 3). Sendo fechado o diagnóstico de papiloma escamoso.

Figura 3: Fotomicrografia mostrando aspecto histopatológico da lesão

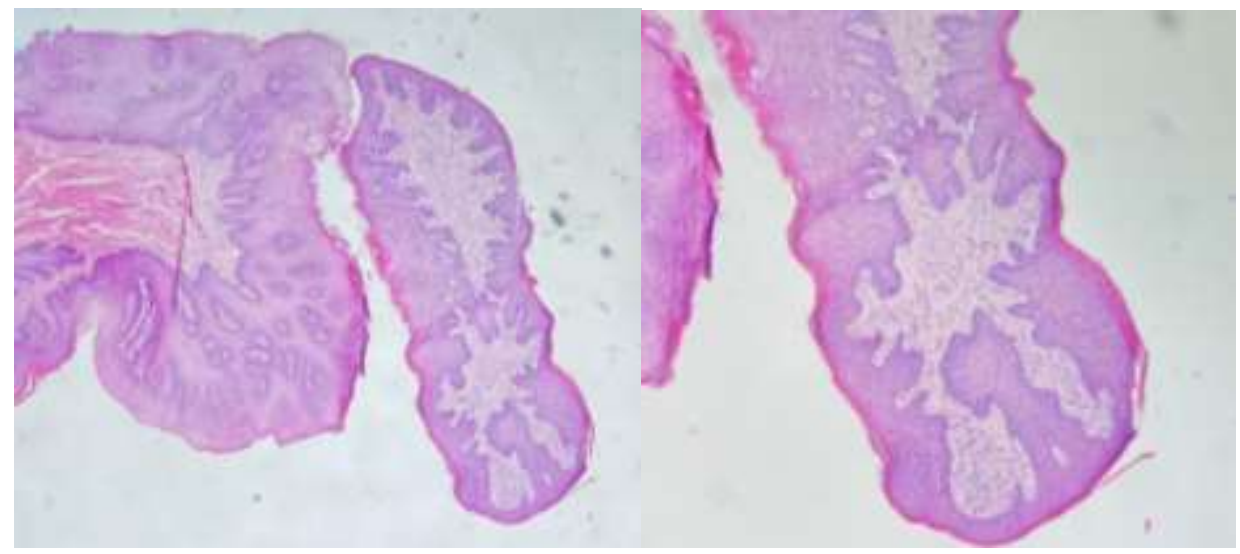

Fonte: Autores.

Realizada orientação junto à família em relação a necessidade de acompanhamento periódicos com o cirurgião dentista para preservação e identificação de futuras possíveis lesões orais.

A paciente foi encaminhada ao médico Ginecologista, já que a mesma nunca havia ido ao especialista, tinha ciclos menstruais regulares e segundo a mãe a filha não havia iniciado a vida sexual. Ao ser questionada sobre a vacina contra o HPV a mãe relatou que a paciente já havia sido imunizada.

Em sua consulta de retorno após uma semana foi observada boa recuperação da área sem sinais de recorrência (Figura 4). 
Figura 4: Aspecto clínico após uma semana da biópsia excisional.

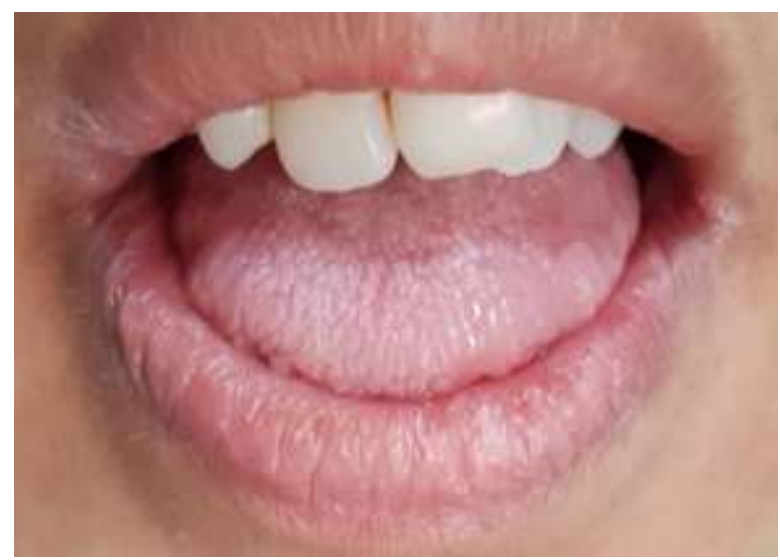

Fonte: Autores.

O presente relato de caso foi autorizado pela cuidadora através da assinatura do Termo de Consentimento Livre e Esclarecido (TCLE).

\section{Discussão}

O HPV atua nas células basais do epitélio escamoso, principalmente em áreas de mucosa, como oral e genital (Ferraro, 2011). A transmissão do HPV pode ser desencadeada mediante ao contato direto com áreas de mucosa infectadas, como durante o parto (horizontal) ou por contato sexual desprotegido (Neville., 2016; Andrade et al., 2019). Neste caso, a progenitora nega início da atividade sexual da filha, foi dada as orientações a família da necessidade de acompanhamento com o ginecologista. A coexistência de lesões em boca e região genital é comum, logo o aconselhamento e acompanhamento da equipe multidisciplinar, com encaminhamento para ginecologista é necessário, para integrar a saúde da paciente de modo conjunto (Silva., 2011).

As lesões associadas ao HPV possuem características clínicas muito semelhantes, o que dificulta seu diagnóstico apenas clínico, especialmente em estágios precoces. Logo, exames complementares precisos auxiliam no diagnóstico conclusivo, como o exame anatomopatológico, hibridização in situ, imuno-histoquímica, microscopia eletrônica, PCR (Reação em Cadeia da Polimerase) e imunofluorescência (Shin et al., 2010; Lane et al., 2006; Leite et al., 2008).

Para o diagnóstico das lesões por HPV normalmente se utiliza o exame clínico e o histopatológico. Achados microscópicos como projeções digitiformes do epitélio superficial, bem como acantose e hiperqueratose auxiliam no diagnóstico (Almeida., 2016). Sendo assim, sempre que o cirurgião dentista se depara com uma lesão que mimetize as características clínicas de um grupo diverso de patologias, como a reportada no nosso relato de caso, é imprescindível que a mesma seja encaminhada para o serviço de patologia. Desta forma, associando o exame clínico e histopatológico, erros diagnósticos podem ser amenizados, o que permite direcionar condutas de tratamento mais precisas e prognósticos mais efetivos (Aquino et al., 2010).

O diagnóstico precoce dessas lesões é fundamental para estabelecer um tratamento eficaz (Testi et al., 2016), quando não houver regressão da lesão, a remoção da mesma é o tratamento (Andrade et al., 2019). Técnicas cirúrgicas com bisturi convencional ou laser de alta potência, assim como o uso da crioterapia e terapia fotodinâmica antimicrobiana são indicados para a remoção da lesão (Orrú, et al., 2019). Não se pode garantir a erradicação definitiva do vírus (Andrade et al., 2019), por isso que retornos periódicos ao dentista são necessários para controle das lesões, já que existe possibilidade de aparecimento de novas lesões ou recidiva da lesão. 
Research, Society and Development, v. 11, n. 1, e3411124292, 2022

(CC BY 4.0) | ISSN 2525-3409 | DOI: http://dx.doi.org/10.33448/rsd-v11i1.24292

Se sabe do potencial oncogênico do HPV, sendo o de alto risco o tipo 16 e 18, tem a capacidade de inserir fragmentos específicos do seu DNA, os genes E6 e E7, no genoma das células infectadas (Shaikh et al., 2015). A associação entre o câncer de orofaringe, anogenital e de colo de útero com o papilomavírus humano está bem estabelecida (Araldi et al., 2018), porém o envolvimento desse vírus no desenvolvimento de Carcinoma de Células Escamosas Oral (CCEO) ainda é controverso. Em 2006 a Food and Drug Administration (FDA) e a Agência Nacional de Vigilância Sanitária (Anvisa) aprovaram a vacina quadrivalente para ser utilizada nos programas preventivos de combate ao câncer de colo de útero relacionados ao papilomavírus humano (Zardo et al., 2014; Pomfret et al., 2011).

Mais de 70 países disponibilizam vacinação gratuita para meninas de 9 a 17 anos de idade (Candotto et al., 2017), visando com isso prevenir estas antes mesmo de iniciarem sua vida sexual ou serem expostas a possíveis infecções do HPV (Faria et al., 2021). As vacinas por si só não podem erradicar ou inibir os vírus, por isso que a eficácia da vacinação contra o HPV é maior em indivíduos que ainda não foram expostos ao vírus, pessoas já infectadas a sua eficácia pode não ser tão notável (Miltz et al., 2014). A vacinação contra o HPV é eficiente em prevenir infecção pelo HPV não apenas no colo de útero, mas também anal e oral (Zhang et al., 2021; Zardo et al. 2014; da Silva et al., 2020).

Desta forma, cabe aos profissionais da odontologia medidas de aconselhamento da importância da vacinação contra o HPV, além de visitas regulares ao dentista para rastreamento de lesões orais iniciais.

\section{Conclusão}

Salienta-se que todas as lesões de boca persistente devem ser biopsiadas, pois lesões que aparentemente inofensivas podem ter diagnóstico que exigem uma abordagem mais criteriosa. Com relação ao tratamento de lesões relacionadas ao HPV, independentemente do tipo, devem ser removidas totalmente. Salientando a importância do tratamento multidisciplinar e orientações dos pais da necessidade do acompanhamento ginecológico e odontológico para garantir uma proservação no estado de saúde bucal. Ressalta-se, que mais estudos devem ser desenvolvidos a despeito da temática aqui abordada, visando com isso obter mais informações e achados sobre o papiloma escamoso em adolescente com deficiência intelectual.

\section{Referências}

Andrade, S. A., Pratavieira, S., Paes, J. F., Ribeiro, M. M., Bagnato, V. S., \& Varotti, F. P. (2019). Oral squamous papilloma: a view under clinical, fluorescence and histopathological aspects. Einstein (Sao Paulo, Brazil), 17(2), eRC4624. https://doi.org/10.31744/einstein_journal/2019RC4624

Araldi, R. P., Sant'Ana, T. A., Módolo, D. G., de Melo, T. A., Spadacci-Morena, D. D., de Cassia Stocco, R., Cerutti, J. M., de Souza, E. B. (2018). The human papillomavirus (HPV)-related cancer biology: An overview. Biomedicine \& Pharmacotherapy, 106(), 1537-1556. 10.1016/j.biopha.2018.06.149

Betz, S. J. (2019). Lesões papilares da mucosa oral relacionadas ao HPV: uma revisão. Patologia de cabeça e pescoço. 13(1), 80-90.

Candotto, V., Lauritano, D., Nardone, M., Baggi, L., Arcuri, C., Gatto, R. et al. (2017). HPV infection in the oral cavity: epidemiology, clinical manifestations and relationship with oral cancer. Oral Implantol (Rome). 2017;10(3):209-20.

Castro, T. M. P. G., Neto, C. E. R., Scala, K. A., et al. (2004). Manifestações orais associada ao papilomavírus humano (hpv) conceitos atuais: revisão bibliográfica. Rev Bras de Otor. 2004; 70(4):546-550.

da Silva, BF, Rodrigues, AR, Freitas, AGP, Vilela, BP, Cavalcante, DR, da Silveira, ELV, ... \& de Moura Vilela, EF (2020). O impacto da adesão vacinal contra o HPV entre jovens: um estudo transversal. Revista Brasileira de Desenvolvimento, 6 (8), 59086-59092.

Faria, A. J. V., Barroso, A. C. F., Lacerda, A. P. S., Mendes, B. M. C., Partata, C. E., Araújo, C. L. de, Santos, F. C. dos, Freitas, M. E. M. A., Moreira, M. V. A., \& Cabral, A. C. G. (2021). HPV: a importância da vacinação para redução do surgimento de lesões pré-malignas do câncer de colo uterino. Revista Eletrônica Acervo Saúde, 13(4), e6946. https://doi.org/10.25248/reas.e6946.2021

Ferraro, C. T. L. et al. (2011). Infecção oral por HPV e lesões epiteliais proliferativas associadas. Jornal Brasileiro de Patologia e Medicina Laboratorial. 47(4), 451-9.

Gleason, A. G., Ponce, D. M. S. \& Gaspar, D. V. (2016). Diagnóstico y tratamiento de un papiloma solitario de lengua. Reporte de caso y revisión de la literatura. Revista odontológica mexicana. 20(1), 39-43 
Research, Society and Development, v. 11, n. 1, e3411124292, 2022

(CC BY 4.0) | ISSN 2525-3409 | DOI: http://dx.doi.org/10.33448/rsd-v11i1.24292

González, G. A., González, P. D. M. S., Vera, G. D. (2016). Diagnóstico y tratamiento de un papiloma solitario de lengua. Reporte de caso y revisión de la literatura. Rev Odont Mex. 20(1):39-43

Miltz, A., Price, H., Shahmanesh, M., Copas, A., Gilson, R. (2014). Systematic review and meta-analysis of L1-VLP-based human papillomavirus vaccine efficacy Against anogenital pre-cancer in women with evidence of prior HPV exposure. PloS One 2014;9:e90348. https://doi.org/10.1371/journal.pone.0090348.

Neville, B. W., Damm, D., Allen, C. M., Bouquot, J. E. (2016). Patologia epitelial. In: Patologia oral \& maxilofacial. (4a ed.), Elsevier Saunders.

Orenuga, O. O., Oluvo, A., Oluwakuyide, R. T., Olawuyi, A. B. (2018). Recurrent oral squamous papilloma in a pediatric patient: Case report and review of the literature. Niger J Clin Pract. 21(12):1674-1677.

Orrù, G., et al. (2019). Infecção por vírus do papiloma humano oral: uma visão geral do diagnóstico e tratamento clínico-laboratoriais." (2019): 81488157.Andrade SA, Pratavieira S, Paes JF, et al. Papiloma escamoso oral: uma visão sob aspectos clínicos, de fluorescência e histopatológicos. Einstein 2019; 17(2):1-4

Pereira A. S., et al. (2018). Metodologia da pesquisa científica. UFSM.

Piña, A. R., et al (2019). Lesões epiteliais orais benignas - associação com papilomavírus humano. Medicina oral, patologia oral y cirugia bucal, 24 (3), e290.

Pomfret, T. C., Gagnon, J. M., Gilchrist, A. T. (2011). Quadrivalent human papillomavirus (HPV) vaccine: a review of safety, efficacy, and pharmacoeconomics. Journal of clinical pharmacy and therapeutics 2011;36(1):1-9.

Shaikh, M. H., Mc, M., Nigel, A. J., Johnson, N. W. (2015). HPV-associated head and neck cancers in the Asia Pacific: A critical literature review \& metaanalysis. Cancer Epidemiology, 39(6), 923-938. 10.1016/j.canep.2015.09.013

Testi, D., Nardone, M., Melone, P., et al. (2016). HPV and oral lesions: preventive possibilities, vaccines and early diagnosis of malignant lesions. Oral Implantol. 2016; 8(2-3):45-51

Yoshida, W. B. (2007). Redação do relato de caso.

Zardo, G. P., Farah, F. P., Mendes, F. G., Franco, C. A. G. D. S., Molina, G. V. M., Melo, G. N. D., \& Kusma, S. Z. (2014). Vacina como agente de imunização contra o HPV. Ciência \& Saúde Coletiva, 19, 3799-3808.

Zhang, J., Qin, Z., Lou, C., Huang, J., Xiong, Y. (2021). The efficacy of vaccination to prevent human papilloma viruses infection at anal and oral: a systematic review and meta-analysis. Public Health. 196:165-171. 10.1016/j.puhe.2021.05.012. 